\title{
In-Situ Deuterium Charging for Direct Detection of Hydrogen in Vanadium by Atom Probe Tomography
}

\author{
S. Dumpala ${ }^{1}$, D. Haley ${ }^{2}$, S.R. Broderick ${ }^{1}$, P.A.J. Bagot ${ }^{2}$, M. P. Moody ${ }^{2}$, and K. Rajan ${ }^{1}$ \\ 1. Department of Materials Science and Engineering and Institute for Combinatorial Discovery, Iowa \\ State University, 2220 Hoover Hall, Iowa State University, Ames, USA. \\ 2. Department of Materials, University of Oxford, Parks Road, Oxford OX13PH, UK
}

The study of hydrogen embrittlement is of great interest for several decades, owing to the large reduction in maximum elongation of hydrogen exposed materials [1]. Towards this end, we will be studying the hydrogen interaction with material microstructure. Even though there exist several competing theoretical models, providing a mechanism by which dislocations and cracks are expedited through microstructures [2], there is a lack of experimental evidence to support differing theoretical models, owing to two main limitations. Firstly, hydrogen is weakly interacting with many radiations (e.g. x-ray, electron), and thus is difficult to image. Secondly, the interactions are truly atomistic, and thus require a scale-matched imaging methodology. Thus there exists a need for real-space quantitative analysis for identification of $\mathrm{H}$, at these nano scales. Such information would allow for greater understanding of the dislocation $\mathrm{H}$ interaction.

With the high chemical sensitivity of Atom Probe Tomography (APT) along with its 3D visualization capabilities, APT is able to overcome these limitations inherent in other methods. Existing challenges in this, owing to hydrogen's high diffusivity, and low solubility in iron-based materials are to maximize signal when charging with $\mathrm{D}_{2}$ when undertaking controlled in-situ $\mathrm{D}_{2}$ charging experiments [3].

In the present work, the existing environmental chamber, which is integrated with the LEAP [4] with a maximum operating pressure range of 50 milli Torr was modified (Fig 1) to allow for atmospheric pressure ( $\sim 1 \mathrm{Atm})$ charging and to be able to use $\mathrm{D}_{2}$ as a feed gas as higher pressure assists in greater deuterium concentration in the material due to theoretical solubility partitioning. Preliminary in-situ deuterium charging experiments performed on the pre-cleaned vanadium samples clearly indicated the significant detection of $\mathrm{D}_{2}$ (Fig. 2). Thus the existing reaction cell design offers several advantages over ex-situ techniques. Firstly the time requirements for transfer can be reduced, owing to the naturally dry (and thus clean) environment, and the presence of a 3-stage vacuum system. Secondly, the existing heating stage allows for hydrogen-1 (protium) to be expelled from the material prior to deuterium charging.

\section{References:}

[1] H. Sugimoto et al, Acta Materiala, 67 (2014) 418.

[2] J. Song et al, Acta Materiala 59 (2011) 1557.

[3] D. Haley et al, International Journal of Hydrogen Energy 39 (2014) 12221

[4] S. Dumpala et al, Ultramicroscopy 141 (2014) 16 
[5] The authors acknowledge the support from Air Force Office of Scientific Research grants: FA955010-1-0256, FA9550-11-1-0158 and FA9550-12-1-0456; and NSF grants: ARI Program CMMI-09389018 and PHY CDI-09-41576.

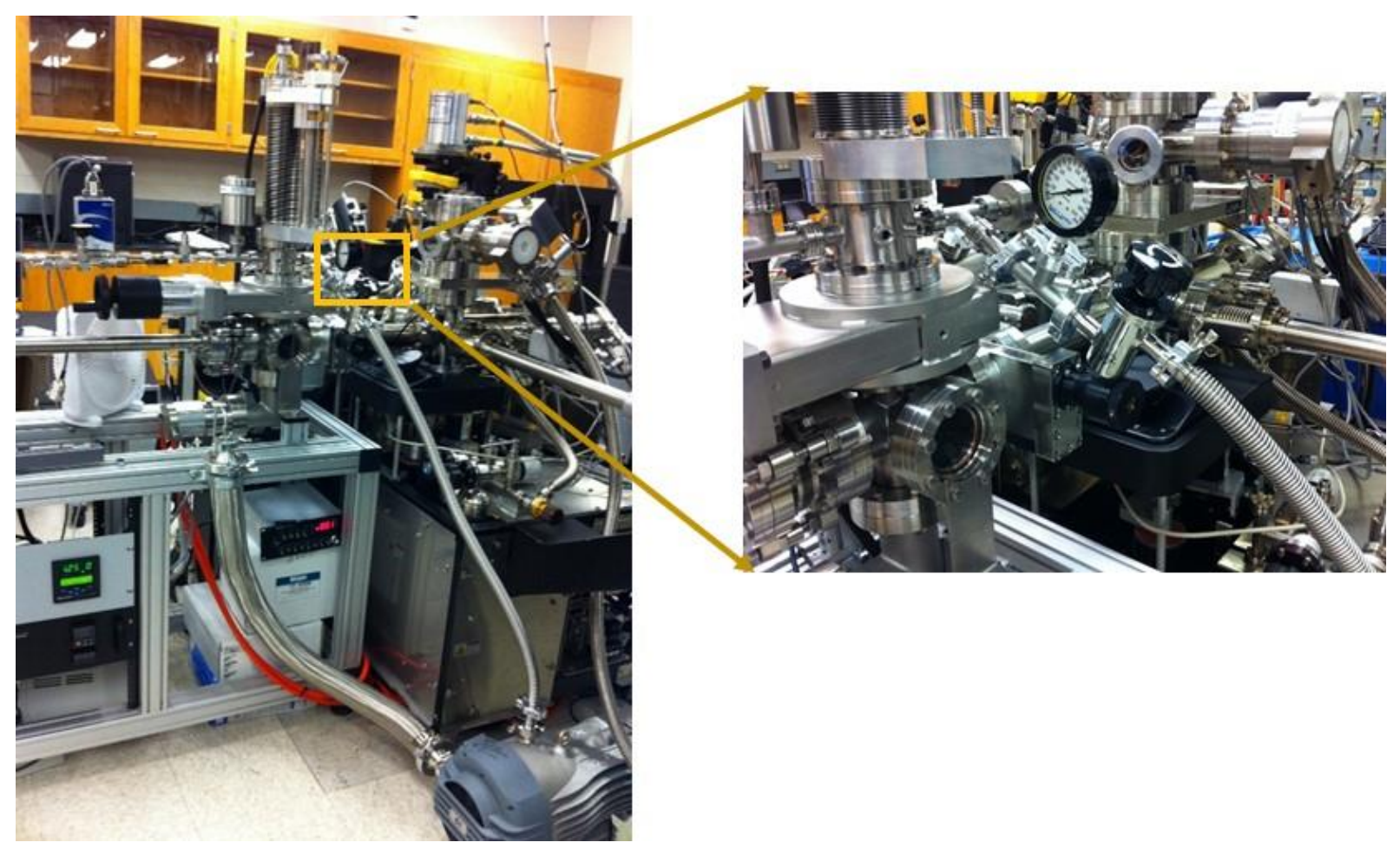

Figure 1. Environmental chamber modified with additional pressure components. Inset shows the close up of the pressure gauge.

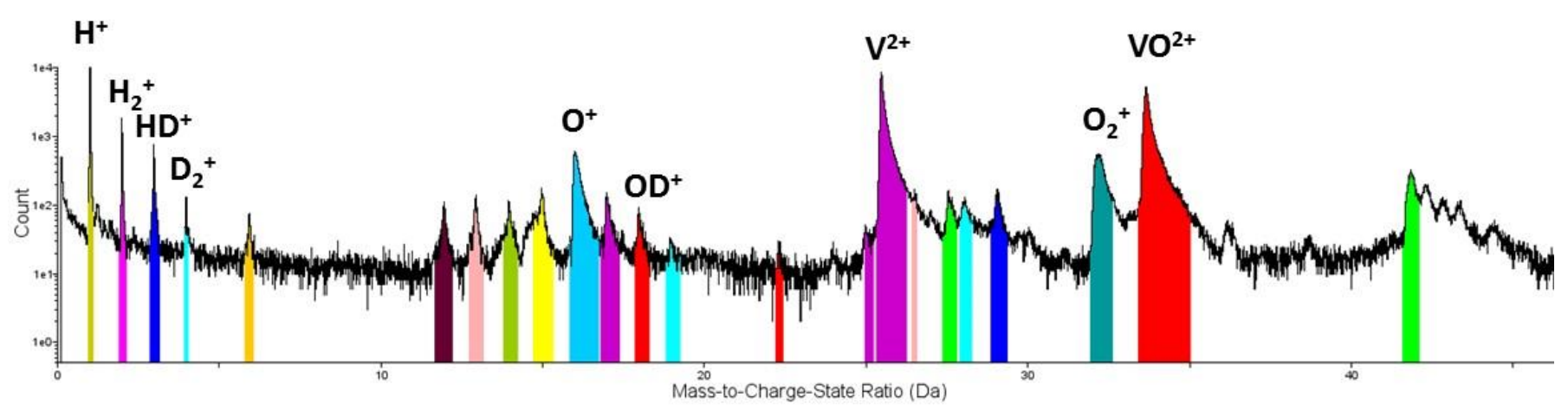

Figure 2. Atom probe tomography mass spectra of the vanadium sample after the in-situ $\mathrm{D}_{2}$ charging, clearly indicating the direct detection of deuterium with presence of peaks corresponding to various deuterium compounds. 